\title{
Coexistence of gastric multiple neuroendocrine tumors with unusual morphological features and gastric signet-ring cell carcinoma
}

\begin{abstract}
Nesrin Uğraș ${ }^{1}$ (D), Pınar Sarkut² (D), Ömer Yerci ${ }^{1}$ (D) Ersin Öztürk² (D)
ABSTRACT The coexistence of signet-ring cell carcinoma and neuroendocrine tumors is very rare. We report a 57-year-old man who presented with a history of weight loss and nausea. Gastric mucosal biopsies obtained during gastrointestinal endoscopy revealed a gastric signet-ring cell carcinoma. The patient underwent a total gastrectomy with a standard D2 lymph node dissection. Ten individual tumors were detected in the resected specimen. Based on the histopathological and immunohistochemical findings, the final diagnosis was co-existing signet-ring cell carcinoma and neuroendocrine tumor. Spindle-shaped cells and extracytoplasmic mucin were noted in some tumor cells forming the neuroendocrine component. This case is a rare synchronous tumor because of its unusual neuroendocrine component.
\end{abstract}

Keywords: Synchronous, gastric, mucin-producing, spindle, neuroendocrine tumor
ORCID IDs of the authors: N.U. 0000-0003-0127-548X; P.S. 0000-0002-2378-0666; 0.Y. 0000-0001-7118-5258; E.0. 0000-0001-8593-5101

Cite this paper as: Uğraş N, Sarkut P, Yerci 0, Öztürk E. Coexistence of gastric multiple neuroendocrine tumors with unusual morphological features and gastric signetring cell carcinoma. Turk J Surg 2018; 34: 152-154.

'Department of General Surgery, Uludağ University School of Medicine, Bursa, Turkey

${ }^{2}$ Department of Surgical Pathology, Uludağ University School of Medicine, Bursa, Turkey

\section{Corresponding Author} Nesrin Uğraş

e-mail:nesrin_ugras@yahoo.com

Received: 09.07.2015

Accepted: 19.08.2015

Available Online Date: 03.01.2018

C) Copyright 2018

by Turkish Surgical Association

Available online at

www.turkjsurg.com

\section{INTRODUCTION}

The coexistence of signet-ring cell carcinoma (SRCC) and neuroendocrine tumor (NET) is very rare (1). SRCC is a histological type in which more than $50 \%$ of the isolated tumor cells contain intracellular mucin (2). Gastric NETs originate from the neuroendocrine cells that are dispersed throughout the stomach, and constitute a very heterogeneous group clinically and pathologically. There is scant literature on synchronous tumors of the stomach that combine SRCC and NET with the presence of extracytoplasmic mucin and spindle-cell morphology. We report this case and discuss the potential etiologies and pathological features of this rare presentation.

\section{CASE PRESENTATION}

A 57-year-old man with a history of chronic atrophic gastritis with complete intestinal metaplasia and hypertension presented with a 2-month history of weight loss and nausea. His physical examination and laboratory results were within normal limits. Upper gastrointestinal endoscopy showed mucosal ulceration and edema in the corpus. Multiple mucosal biopsies revealed gastric SRCC histologically. Computed tomography of the abdomen showed circular thickening of the corpus wall. The patient underwent a total gastrectomy with a standard D2 lymph node dissection. The resected specimen contained 10 different tumors. One was a centrally deep ulcerated mass $(1.2 \times 0.7 \times 0.5 \mathrm{~cm})$ and the other nine were exophytic, tan-colored polypoid masses (diameter of the largest polyp was $1.1 \times 1.1 \mathrm{~cm}$ ) that were similar in appearance to a submucosal tumor at the corpus of the stomach. Pathologically, the ulcerated mass was a gastric SRCC that arose from the gastric mucosa and invaded the submucosa (Figure 1). The tumor cells of the SRCC were positive for pancytokeratin, epithelial membrane antigen, and Ki67 (70\% of labeled cells). Histopathologically, the other nine polypoid tumors demonstrated polygonal, round, spindle-shaped features with vesicular nuclei cells arranged in trabecular, nested, and plexiform patterns in the submucosa and muscular layer, suggesting neuroendocrine cell proliferation (Figure 2). No necrosis or mitosis was observed. The tumor cells of the polypoid tumors were positive for pancytokeratin, synaptophysin, chromogranin A, CD57, protein gene protein 9.5 (PGP9.5), and Ki67 (2\% of labeled cells) (Figure 3).

Immunostaining for mucins indicated that the NET cells expressed MUC5AC and MUC1. These cells were negative for gastrin, serotonin, p53, CD34, CD117, and vimentin. To investigate the presence of neuroendocrine differentiation of SRCC tumor cells, an immunohistochemical study was performed. The signet-ring cells were negative for synaptophysin, chromogranin A, and CD57 (PGP9.5). Spindle-shaped cells and extracytoplasmic mucin were noted in some tumor cells of the neuroendocrine component. Mucicarmine staining of the neuroendocrine cells with a different morphology showed luminal positivity; in contrast, the cytoplasm of the SRCC tumor cells stained for mucicarmine (Figure 3). There was no transition between SRCC and NET, and the latter contained mucin-producing, spindle-shaped cells. These histopathological and immunohistochemical findings led to a final diagnosis of a collision tumor composed of a SRCC and NET (Grade 1, according to the WHO 2010 criteria) of the stomach. No 


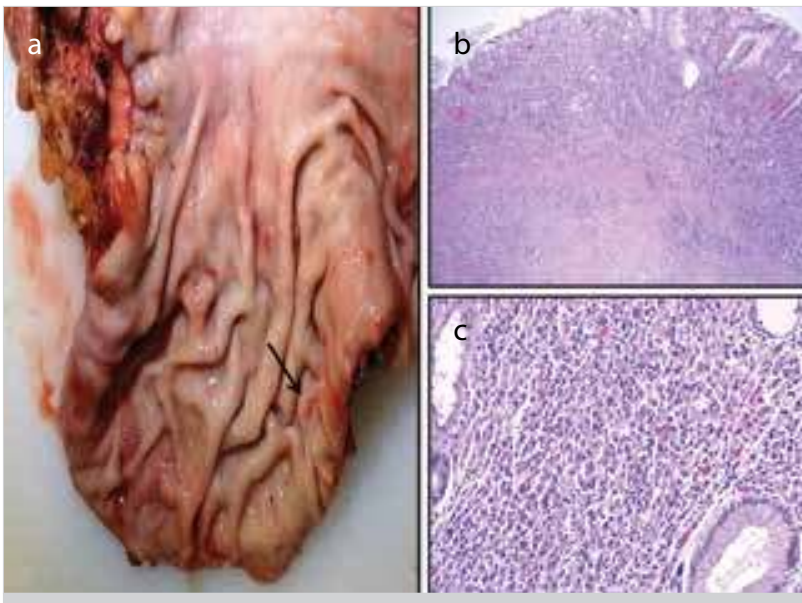

Figure 1. a-c. (a) Grossly, the resected specimen was a tanred tumor mass with ulceration in the stomach (arrow), (b) Microscopically, the ulcerated mass was signet-ring cell adenocarcinoma ( $\times 200$, H\&E stain), (c) Signet-ring cell carcinoma cells with eccentric nuclei and abundant cytoplasm ( $\times 400, \mathrm{H} \&$ E stain)
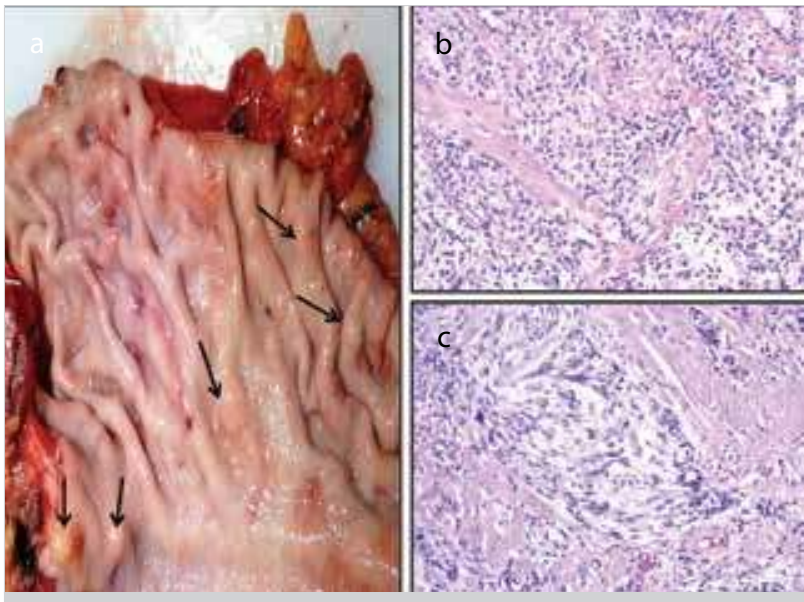

Figure 2. a-c. (a) Grossly, the resected specimen comprised tan-yellow polypoid masses (arrow), (b) Microscopically, the polypoid masses contained uniform populations of small cells with a trabecular, solid growth pattern ( $\times 400$, H\&E stain), (c) Cords of neuroendocrine cells with a spindle shape, mucinous matrix, and a more diffuse area of sheet-like growth

lymphatic permeation, vascular invasion, or perigastric lymph node involvement was observed for either component. The adjacent gastric mucosa showed chronic active gastritis with prominent complete intestinal metaplasia. No Helicobacter pylori was detected.

Postoperatively, the patient recovered uneventfully and had no surgical complications. The patient was treated with adjuvant chemotherapy. Follow-up after one year showed no evidence of recurrence or metastasis. A signed written informed consent was taken from the patient.

\section{DISCUSSION}

The incidence of gastric NET has increased with the development of imaging modalities and increased awareness. SRCC is an unfavorable subtype of gastric carcinoma that may require more aggressive treatment, and which typically lacks cell cohesion and, therefore, expands in an infiltrative pattern (2).

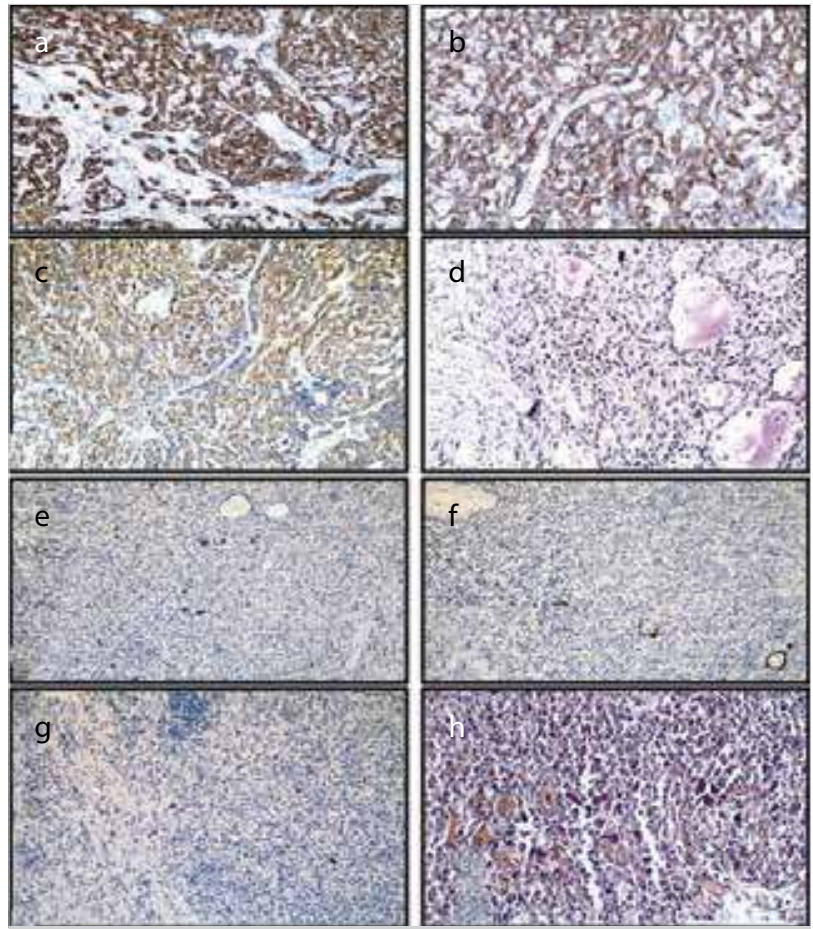

Figure 3. a-h. Immunohistochemical and histochemical findings. (a-c) Neuroendocrine tumor cells expressed PGP9.5, CD57, and synaptophysin, (d) Mucicarmine staining showed focal luminal positivity in the NET component, (e-g) Signet-ring cell carcinoma component cells did not stain for PGP9.5, CD57, or synaptophysin, (h) Mucicarmine staining showed epithelial mucin within the cytoplasmic vacuoles of the signet-ring cells, (a-h) Magnification, $\times 400$

The 2010 World Health Organization (WHO) classification classifies gastric NETs into the following five categories: 1) NET Grade 1, well-differentiated endocrine tumor; 2) NET Grade 2 ; 3) neuroendocrine carcinoma; 4) mixed adenoneuroendocrine carcinoma; and 5) hyperplastic and preneoplastic lesions (3). NET G1 tumors are composed of small-to-medium-sized round-to-polygonal cells with scant-to-moderate amounts of eosinophilic cytoplasm with an organoid, trabecular pattern (3). In this case, all of the NETs were evaluated as Grade 1 according to the WHO 2010 criteria. Various morphological features similar to those presented here can be observed in NETs, such as spindle-shaped cells, acinar cells, signet-ring cells, and mucin-producing cells (goblet cells) $(4,5)$. The presence of mucin in the neuroendocrine component is reminiscent of goblet cell carcinoid. Goblet cell carcinoid is a special type of neuroendocrine tumor that usually occurs in the appendix, but may occasionally be present in other gastrointestinal organs (6). Gui defined the gastric localization of goblet cell NETs (6). Unlike normal NETs, these tumors contain co-existing mucinladen goblet-shaped cells and neuroendocrine cells arranged in nests or clusters, with weak or partial immunopositivity for neuroendocrine markers (7). The mucin in these tumors tends to be intracytoplasmic $(5,6)$. In our case, the mucin was extracytoplasmic and there were no goblet cells. The neuroendocrine cells with extracytoplasmic mucin and a spindle cell morphology may be a new cell variant in gastric NETs.

In a recent study, Domori et al. (8) investigated the mucin phenotype in gastric neuroendocrine neoplasms. No cases of NET Grade 1 and Grade 2 expressed the mucin markers MUC5AC, 
MUC6, or MUC2. Conversely, neuroendocrine carcinoma cases had higher rates of positivity for each mucin marker. In contrast, in our case, the NET Grade 1 tumor cells expressed MUC5AC and MUC2.

Synchronous tumors are believed to result from two separate neoplasms at the primary or another site and may behave differently. The tumor histopathological and immunophenotypic features may be discrete or the same with clear demarcation of the two tumors, indicating that this lesion is a synchronous tumor consisting of SRCC and NET morphologically, consistent with mucin-producing cells. Few cases of gastric NETs coexisting with adenocarcinoma have been reported, and only two have involved SRCC (1).

These findings suggest that chromosomal analysis using comparative genomic hybridization could reveal whether the neuroendocrine component is related to SRCC clonally or whether they are in fact unrelated tumors.

\section{CONCLUSION}

This case was a rare example of synchronous tumors because its neuroendocrine component tumor cells contained extracytoplasmic mucin and were spindle-shaped; these attributes are not seen in classic gastric NET Grade 1 tumors.

Informed Consent: Written informed consent was obtained from patient who participated in this study.

Author Contributions: Concept - N.U., Ö.Y.; Design - N.U., Ö.Y.; Supervision - Ö.Y., E.Ö.; Funding - E.Ö.; Materials - N.U., P.S., E.Ö.; Data Collection and/or Processing - N.U., P.S., Ö.Y., E.Ö.; Analysis and/or Interpretation - N.U., Ö.Y., P.S.; Literature Review - N.U., P.S., Ö.Y.; Writer - N.U., Ö.Y.; Critical Review - Ö.Y., E.Ö.; Other - N.U.
Conflict of Interest: The authors have no conflicts of interest to declare.

Financial Disclosure: The authors declared that this study has received no financial support.

\section{REFERENCES}

1. Yang L, Zhang HT, Zhang X, Sun YT, Cao Z, Su Q. Synchronous occurrence of carcinoid, signet-ring cell carcinoma and heterotopic pancreatic tissue in stomach: A case report and literature review. World J Gastroenterol 2006; 12: 7216-7220. [CrossRef]

2. Kwon KJ, Shim KN, Song EM, Choi JY, Kim SE, Jung HK, et al. Clinicopathological characteristics and prognosis of signet ring cell carcinoma of the stomach. Gastric Cancer 2014; 17: 43-53. [CrossRef]

3. Solcia E, Arnold R, Capella C, Klimstra DS, Klöppel G, Komminoth $P$, et al. Neuroendocrine neoplasms of the stomach. In: "WHO Classification of Tumours of the Digestive System" ed. by Bosman FT, Carneiro F, Hruban RH, Theise ND, WHO Press, Lyon 2010; 6468.

4. Tsuta K, Kalhor N, Wistuba I, Moran CA. Clinicopathological and immunohistochemical analysis of spindle-cell carcinoid tumour of the lung. Histopathology 2011; 59: 526-536. [CrossRef]

5. Fujiyoshi Y, Kuhara H, Eimoto T. Composite glandular endocrine cell carcinoma of the stomach. Report of two cases with goblet cell carcinoid component. Pathol Res Pract 2005; 200: 823-829. [CrossRef]

6. Gui X, Qin L, Gao ZH, Falck V, Harpaz N. Goblet cell carcinoids at extraappendiceal locations of gastrointestinal tract: an underrecognized diagnostic pitfall. J Surg Oncol 2011; 103: 790-795. [CrossRef]

7. CM Fenoglio-Preiser, RR Pascal, KH. Perzin. Tumors of intestine, In: H.W. Hartmann, L.H. Sobin (Eds.), Atlas of Tumor Pathology 1990; 2nd series, Fascicle 27, Armed Forces Institute of Pathology, Washington, DC pp. 263-292.

8. Domori K, Nishikura K, Ajioka Y, Aoyagi Y. Mucin phenotype expression of gastric neuroendocrine neoplasms: analysis of histopathology and carcinogenesis. Gastric Cancer 2014; 17: 263-272.[CrossRef] 